\title{
THE TUNNEL ETCHING OF ALUMINIUM
}

\author{
N. F. JACKSON \\ Allen Clark Research Centre, The Plessey Company Limited, Caswell, Towcester, Northants, U.K.
}

(Received April 3, 1974)

\begin{abstract}
The electrochemical potential for the active dissolution of aluminium in sodium chloride solution is found to be linearly related to the logarithm of the chloride ion concentration. The value of $E_{0}$ calculated on the basis of the postulated electrode reaction is approximately $0.3 \mathrm{~V}$ more negative than the experimental value and a similar inequality is observed for bromide and iodide electrolytes.

The electrochemical etching of aluminium in chloride, bromide or iodide solutions is initiated at weak points in the surface oxide film. Subsequent stages in the dissolution process involve a random attack leading to the formation of cavities and a selective attack leading to tunnelling. The dominance of one or other of these dissolution modes is largely dependent upon the electrochemical parameters. It is suggested that the reason for this dependency is associated with an alteration in the electrode potential for dissolution resulting from changes in the halide ion concentration within the tunnel structure.
\end{abstract}

\section{INTRODUCTION}

The electrochemical etching of aluminium foil in a sodium chloride electrolyte is a well established process for enlarging the surface area of electrolytic capacitor electrodes. The use of highly etched electrodes permits a reduction both in costs and in the size of the capacitor. Full advantage may be taken of these factors since the process used to deposit the oxide dielectric, namely anodisation, has an exceptional throwing power.

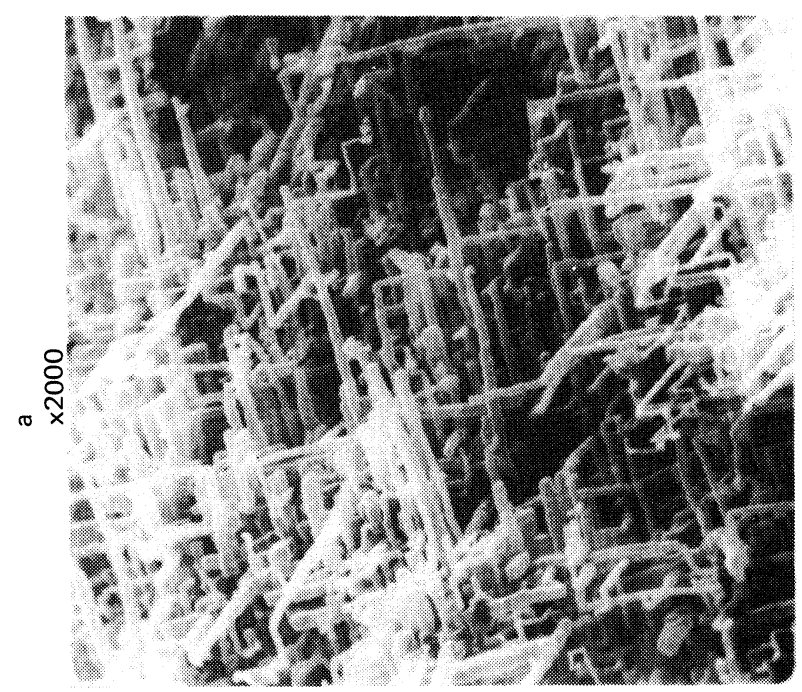

Although a considerable amount of empirical knowledge about electrochemical etching has been accumulated, there is a need to obtain a better understanding of the underlying mechanisms that produce the complex tunnelling patterns observed in etched foils. As an example, the illustrations in Figure 1 show the structure of a foil used for low voltage applications. The tunnels are 10-20 $\mu \mathrm{m}$ long and about $0.25 \mu \mathrm{m}$ wide. Typically, there are in the region of $10^{8}$ tunnels $\mathrm{cm}^{-2}$ of surface and the increase in surface area is about 50 times. The

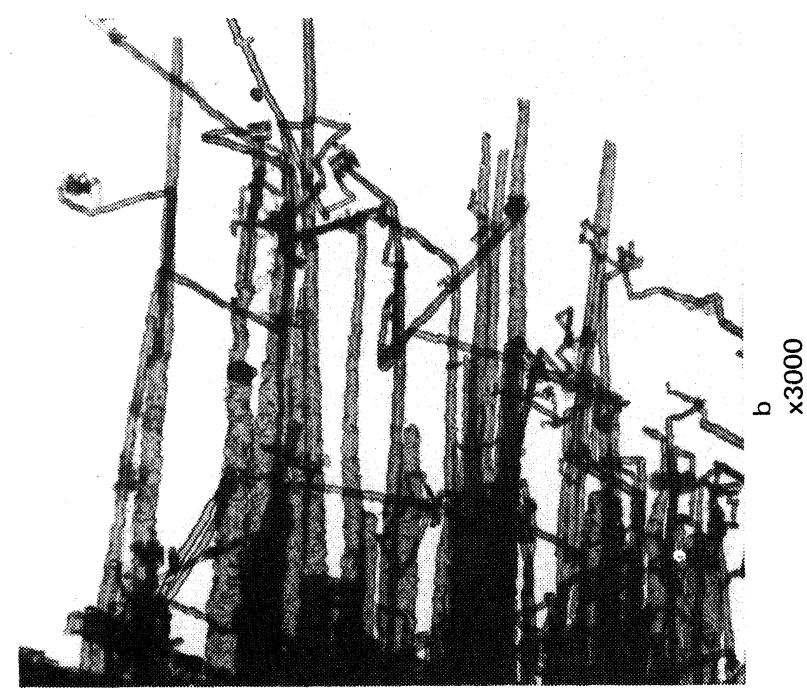

FIGURE 1 Tunnel structure of etched aluminium foil: (a) Scanning electron microscope (b) Transmission electron microscope. 
structural features are important because, as well as controlling the surface area, the morphology largely determines many of the electrical properties of the capacitor, e.g. dissipation factor, performance at low temperature or high frequency, and rated levels of ripple current.

\section{ELECTROCHEMICAL STUDIES}

\subsection{Experimental}

The aluminium foil employed in this work had a purity of $99.99 \%$ and a thickness of $100 \mu \mathrm{m}$. Specimens were cut so as to have a rectangular active area of dimensions $2.0 \times 2.5 \mathrm{~cm}$, the integral tags being stopped off with lacquer. The specimens were precleaned by immersing for 1 minute in 3\% sodium hydroxide at $30^{\circ} \mathrm{C}$ for 60 seconds, followed by rinsing in deionised water.

The electrochemical measurements were made in a glass cell of conventional construction having a closed loop for stirring. The aluminium specimens were suspended centrally between parallel platinum electrodes and electrode potentials were measured by means of a Luggin capillary using a saturated calomel electrode. The solution, which was stirred, was open to the atmosphere. All recorded electrode potentials are given with respect to the standard hydrogen electrode.

Initially an examination was made of the open circuit behaviour of aluminium in various halide electrolytes. Subsequently, the effect of anodic polarisation under galvanostatic and potentiodynamic conditions was investigated.

\subsection{Open Circuit Behaviour}

The change of electrode potential with time when specimens were immersed in $3.42 \mathrm{M}$ sodium chloride solution is shown in Figure 2. For comparison, the behaviour in a typical barrier film forming solution, ammonium pentaborate, is also given. In the penta-

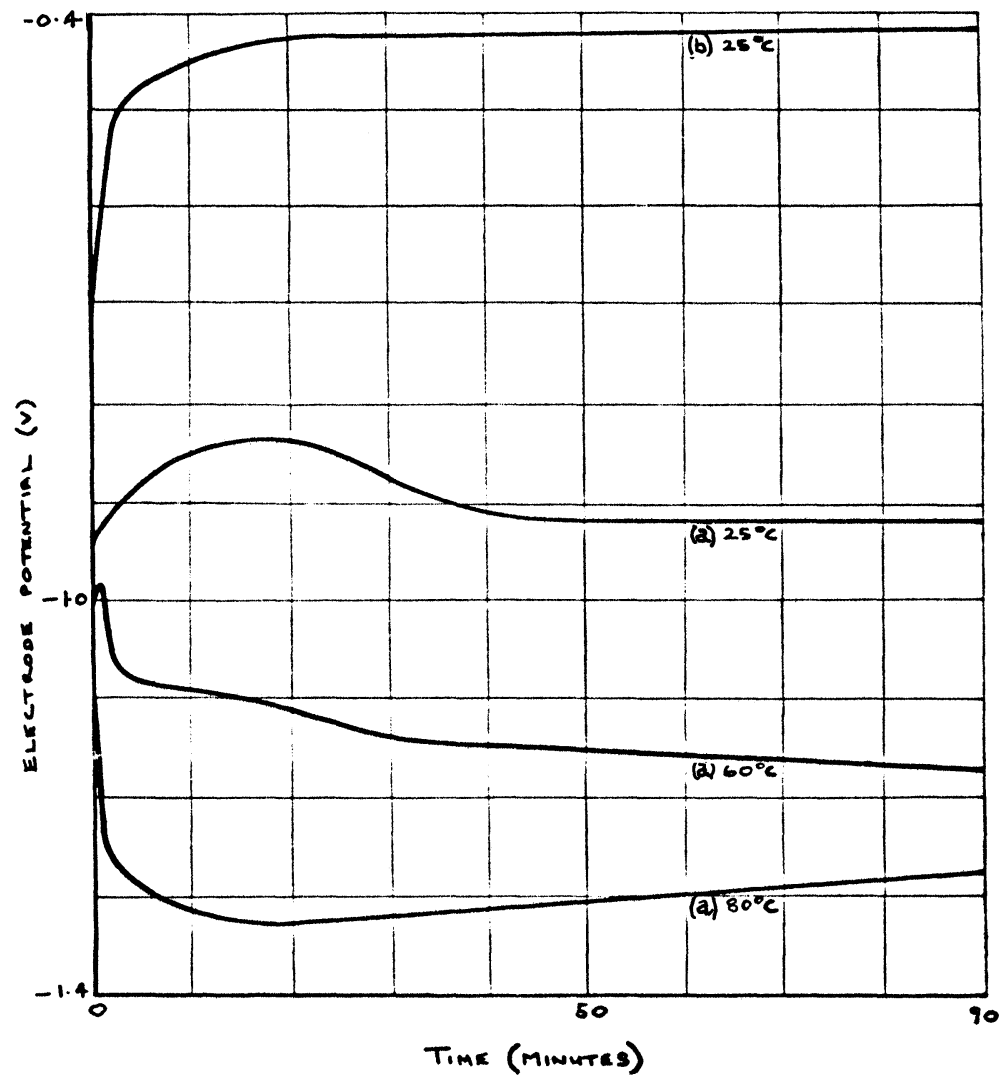

FIGURE 2 Electrode potential of aluminium in: (a) $3.42 \mathrm{M}$ sodium chloride; (b) Ammonium pentaborate (saturated). 
borate solution at $25^{\circ} \mathrm{C}$ the electrode potential increased rapidly to about $-0.5 \mathrm{~V}$, subsequently continuing to increase more slowly. In sodium chloride solution at $25^{\circ} \mathrm{C}$, the potential initially increased, reached a maximum and then fell to more negative values. At higher temperatures, the initial rise was barely perceptible $\left(60^{\circ} \mathrm{C}\right)$, or not observed at all $\left(80^{\circ} \mathrm{C}\right)$, and the final potential was significantly more negative the higher the temperature. In boiling $3.42 \mathrm{M}$ sodium chloride, copious gassing ensued and diffraction studies of the metal surface indicated the formation of boehmite. Similar effects were observed with bromide and iodide electrolytes. As a general rule, the final electrode potential became more negative when the temperature or the halide concentration was increased.

The changes in electrode potential of the aluminium specimens in the ammonium pentaborate solution are consistent with the formation of an anodic oxide layer supported by the cathodic reduction of dissolved oxygen. On the other hand, in a halide solution the anodic reaction produces an hydrated layer and it seems probable that the cathodic reaction is the reduction of water to hydrogen. In partial confirmation of the latter, an examination of the cathodic polarisation of aluminium in $3.42 \mathrm{M}$ sodium chloride at $25^{\circ} \mathrm{C}$ (Figure 3) indicated current densities of $3 \mu \mathrm{A} \cdot \mathrm{cm}^{-2}$ at $-1.0 \mathrm{~V}$ and about $80 \mu \mathrm{A} . \mathrm{cm}^{-2}$ at $-1.3 \mathrm{~V}$.

\subsection{Pitting Potentials}

On the basis of a preliminary investigation involving both galvano-static and potentiostatic techniques, the former was chosen for studying the anodic polarisation of aluminium. The method adopted was to apply a current of $1 \mu \mathrm{A} . \mathrm{cm}^{-2}$ and record the change in electrode potential with time. A typical result is illustrated in Figure 4 for a $1.71 \mathrm{M}$ sodium chloride solution at $25^{\circ} \mathrm{C}$. After a short time interval, the potential established a linear rate of increase with time, a relationship consistent with the formation of a barrier oxide. At a certain potential, the slope reduced to zero and the potential then underwent periodic fluctuations (not shown). Pitting can be observed after a few minutes at this potential, the value of which is taken as the pitting potential $\left(E_{\text {pit }}\right)$. This is the value at which active dissolution first occurred; below the pitting potential, oxide film formation took place.

The above method was used to determine the relationship between $E_{\mathrm{pit}}$ and chloride ion concentration in the range $0.1-1.7 \mathrm{M}$. Problems were encountered at concentrations outside this range as indicated below:

1) $\mathrm{Cl}^{-}>1.7 \mathrm{M}$ At concentrations above $1.7 \mathrm{M}$, because of the dominance of the hydration reaction, it was not possible to control the electrode reaction with an applied current of $1 \mu \mathrm{A} \cdot \mathrm{cm}^{-2}$. In this region

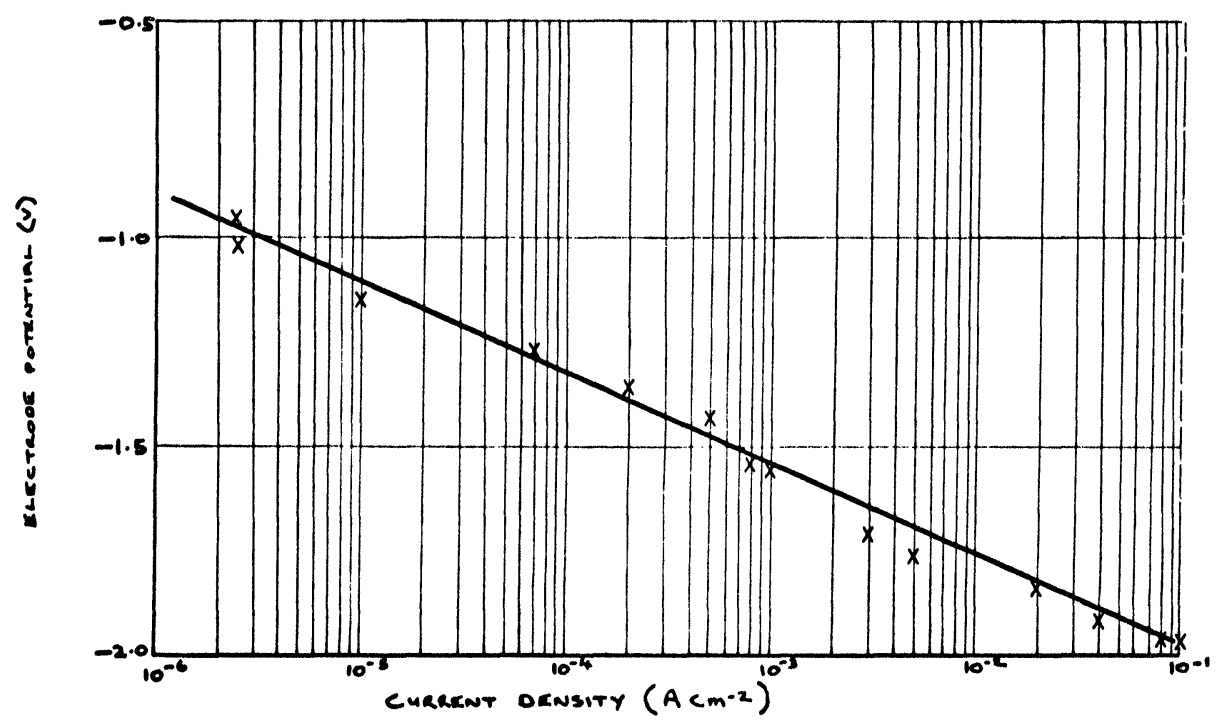

FIGURE 3 Cathodic polarisation of aluminium in $3.42 \mathrm{M}$ sodium chloride at $25^{\circ} \mathrm{C}$. 


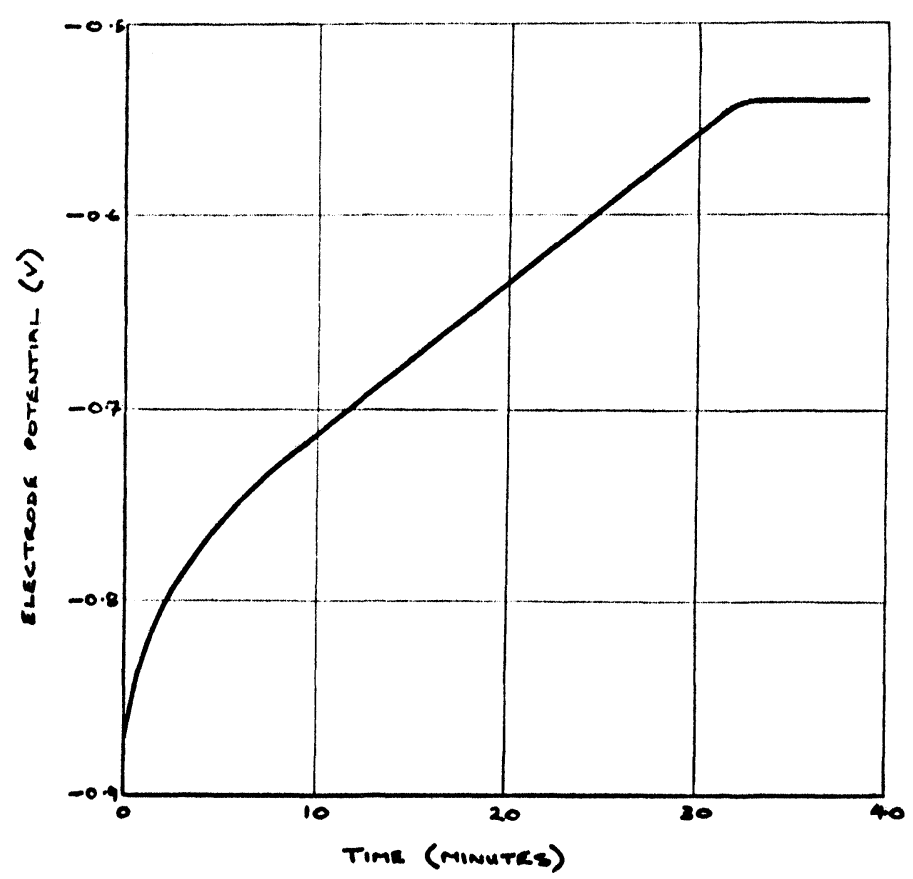

FIGURE 4 Anodic polarisation of aluminium at a current density of $1 \mu \mathrm{A} \mathrm{cm}^{-2}$ in $1.71 \mathrm{M}$ sodium chloride at $25^{\circ} \mathrm{C}$.

two alternative procedures were used. In the first procedure, the current was increased to $5 \mu \mathrm{A} \cdot \mathrm{cm}^{-2}$; in the second, the rate of the hydration reaction was reduced by pre-anodising to $4 \mathrm{~V}$ in an ammonium pentaborate solution and a current of $1 \mu \mathrm{A} \cdot \mathrm{cm}^{-2}$ could then be employed. In practice, both procedures were found to give essentially the same result. This insensitivity of $E_{\mathrm{pit}}$ to the thickness of the oxide layer is in agreement with the work of Bohni and Uhlig. ${ }^{1}$

2) $\mathrm{Cl}^{-}<0.1 \mathrm{M}$ In solutions of concentration less than $0.1 \mathrm{M}$, marked polarisation, apparently caused by concentration changes in the electrolyte close to the metal surface, was observed. However, if the current was substantially reduced below $1.0 \mu \mathrm{A} \cdot \mathrm{cm}^{-2}$ the hydration reaction could not be suppressed. In consequence, in this region of concentration the specimens were pre-anodised to $4 \mathrm{~V}$ and then polarised at $0.1 \mu \mathrm{A} . \mathrm{cm}^{-2}$.

As shown in Figure 5, a linear relationship was observed between $E_{\text {pit }}$ and the logarithm of the chloride ion concentration. The slope of the line is $-0.061 \mathrm{~V}$ and the potential at unit concentration of chloride ions is $-0.52 \mathrm{~V}$. The slope is sufficiently close to $R T / F$ for the relationship to be represented by a Nernst equation of the form:

$$
E_{\text {pit }}=-0.52-R T / F \log \left(\mathrm{Cl}^{-}\right)
$$

The above equation is characteristic of an electrode of the second type, the simplest overall reaction for which may be written as

$$
\mathrm{Al}+3 \mathrm{Cl}^{-}=\mathrm{AlCl}_{3}+3 \mathrm{e}
$$

The $E_{\text {pit }}$ values for bromide and iodide electrolytes were determined at two concentrations only, $0.60 \mathrm{M}$ and $3.42 \mathrm{M}$. Making the assumption of a similar relationship to concentration as that for the chloride ion, values for $E_{0}$ were deduced. These experimental values of $E_{0}$ for the three halides, together with values calculated from free energy data on the basis of Eq. (2) are listed in Table I.

TABLE I

\begin{tabular}{|c|c|c|}
\hline Halide & $\begin{array}{l}\text { Experimental } \\
E_{0} \\
\text { (volts) }\end{array}$ & $\begin{array}{l}\text { Calculated } \\
E_{0} \\
\text { (volts) }\end{array}$ \\
\hline $\mathrm{Cl}^{-}$ & -0.52 & -0.84 \\
\hline $\mathrm{Br}^{-}$ & -0.40 & -0.68 \\
\hline I & -0.23 & -0.55 \\
\hline
\end{tabular}

Experimental and calculated $E_{0}$ values 


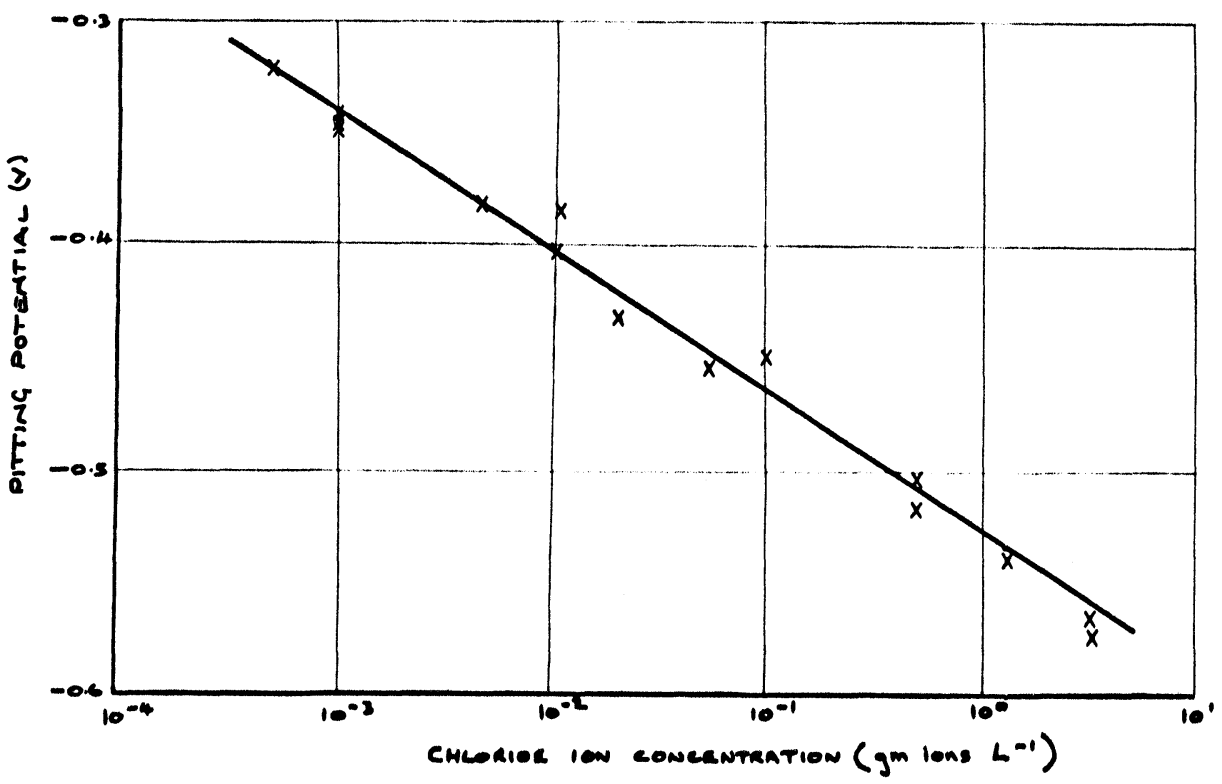

FIGURE 5 Variation of pitting potential with logarithm of chloride ion concentration at $25^{\circ} \mathrm{C}$.

A relatively constant difference of $0.3 \mathrm{~V}$ is apparent between the experimental and calculated values of $E_{0}$.

\subsection{Potentiodynamic Measurements}

The major problem in studying polarisation data lies in establishing the correct value of the current density. Firstly, because of the localised nature of the reaction, the area undergoing dissolution is considerably smaller than the nominal area of the specimen. Secondly, by the very nature of the process, the active area tends to increase as the reaction progresses. There appears to be no simple way of overcoming these difficulties and so in the work described below the current density is related to the total surface area of the specimen.

The general form of the relationship between electrode potential and the logarithm of the current density is shown in Figure 6. In this experiment the potential was cyclically scanned from -1.0 to $0.0 \mathrm{~V}$ at a speed of $33 \mathrm{mV} \cdot \mathrm{sec}^{-1}$ in sodium chloride solution. Rather unexpectedly, repeated cycling showed little deviation from this relationship. However, later work (para. 3) has shown that tunnelling is minimal under these conditions and so changes in surface area may not be very large after the first cycle. A notable feature of Figure 6 is the increase in current density after the potential reversal at $0.0 \mathrm{~V}$. This phenomenon has been discussed by Broli and Holtan $^{2}$ who came to the conclusion that it was due to "a lack of established electrochemical equilibria". Since the hysteresis is closely repeated on all the cycles, it seems unlikely to be associated with a progressive alteration in the surface structure. Most probably the loop can be related to changes in the concentration of the electrolyte during the voltage increasing phase, a change which is subsequently dissipated when the voltage decreases. The average slope between $E_{\text {pit }}$ and $0.0 \mathrm{~V}$ is in the region of $-180 \mathrm{mV}$, a value that is found to decrease quite markedly with scanning speed. For example, at a speed of $1.7 \mathrm{mV} \cdot \mathrm{sec}^{-1}$, the average slope is reduced to $-25 \mathrm{mV}$.

\section{STRUCTURAL STUDIES}

A study of the metal surface in the early stages of the etching process suggests that the initial attack occurs at weak parts in the surface oxide film. These weak points, which permit penetration of halide ions, are probably related to physical and chemical imperfections at the metal surface. In consequence, rolling features and grain boundaries play an important role in determining where the attack is initiated. Depending upon the electrochemical conditions applied, it is often possible to observe these effects (Figure 7). A marked grain boundary delineation is shown in Figure 7(a), whereas both grain boundary and rolling line attack is indicated in Figure 7(b). The 


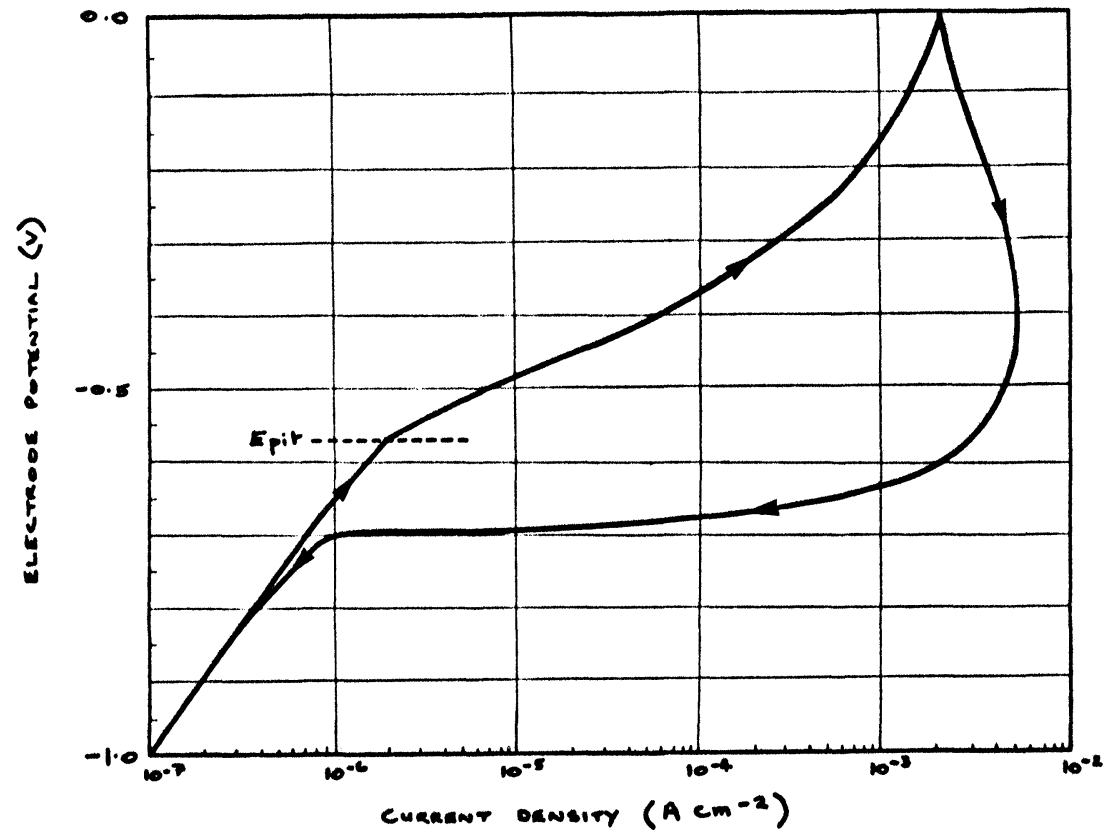

FIGURE 6 Potentiodynamic polarisation of aluminium at a scanning speed of $33 \mathrm{mV} \sec ^{-1}$ in $3.42 \mathrm{M}$ sodium chloride at $20^{\circ} \mathrm{C}$.

pretreatment of the foil prior to etching, e.g. annealing, scratch brushing or chemical pickling, can influence directly the number of sites of attack and this aspect has important commercial considerations.

The study of the structures produced by the

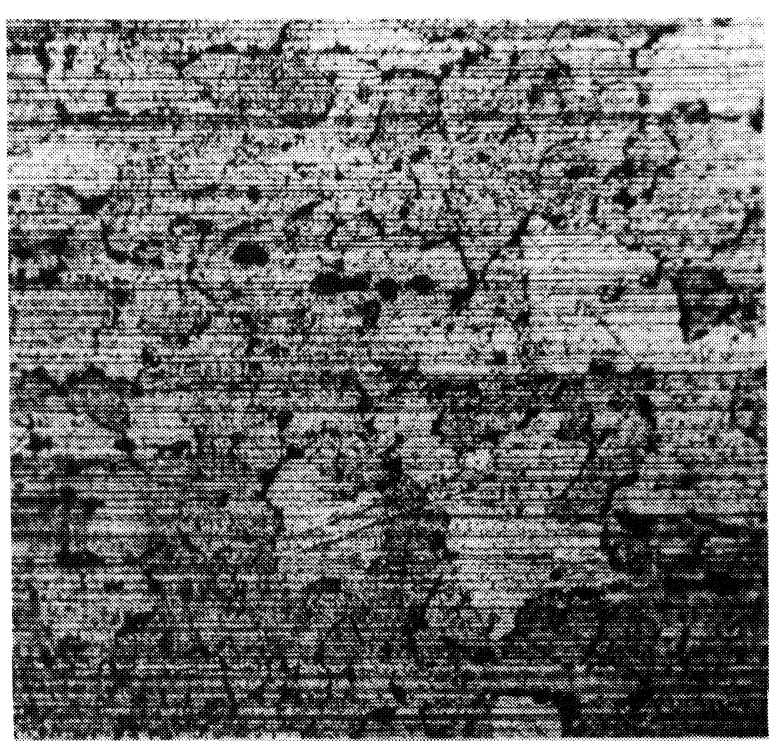

a $\times 150$ etching process below the surface was made by means of oxide replicas in association with the scanning electron microscope $e^{3,4,5}$. These investigations indicated that the attack on the metal shows two distinct features. Firstly, the dissolution is highly

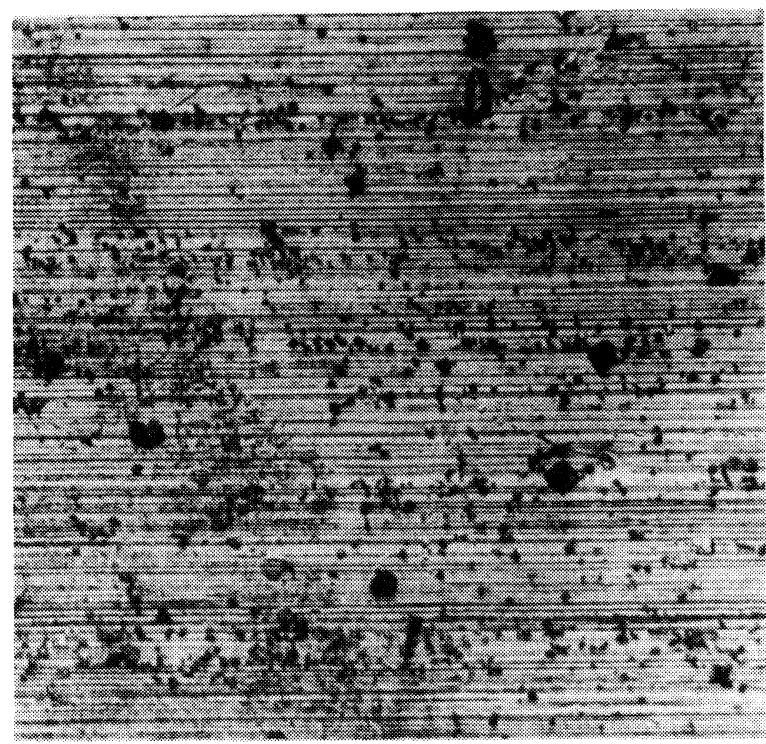

b $\times 150$

FIGURE 7 Initiation of etch pits: (a) $3.42 \mathrm{M}$ sodium chloride, $0.5 \mathrm{~A} \mathrm{~cm}^{-2}, 2$ coulombs $\mathrm{cm}^{-2}, 20^{\circ} \mathrm{C}$; (b) $0.34 \mathrm{M}$ sodium chloride, $0.3 \mathrm{~A} \mathrm{~cm}^{-2}, 2$ coulomb $\mathrm{cm}^{-2}, 20^{\circ} \mathrm{C}$. 

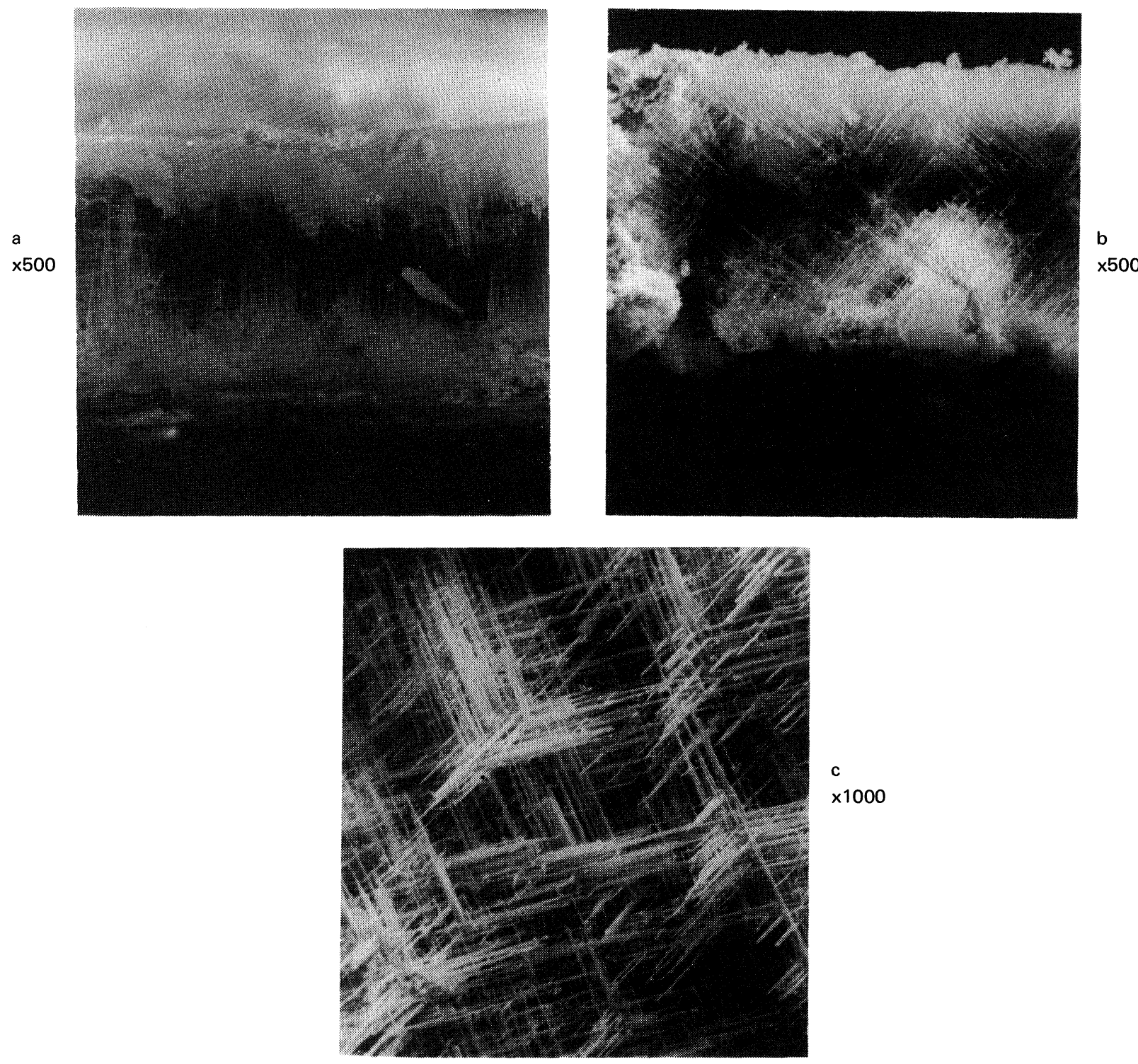

FIGURE 8 Relationship between tunnelling and grain orientation: (a) Etched foil section, $\{100\}$ surface orientation; (b) Etched foil section $\{110\}$ surface orientation; (c) Single crystal $\{111\}$ surface orientation.

directional, being propagated along $\{100\}$ crystallographic planes (Figure 8). Secondly, the structure gives the appearance of resulting from the removal of small cubes from the metal. Furthermore, it appears that the cubes may be removed either randomly or selectively. In the former case, approximately hemispherical cavities are produced in the metal surfaces; in the latter case tunnels having the same cross-sectional area as the cubes grow outwards from the walls of the cavities. These effects are illustrated in Figure 9, which shows the stages in the develop- ment of the tunnel structure in a commercial etching process. The schematic diagram (Figure 10) is drawn on the assumption that the surface grains are orientated with the $\{100\}$ planes uppermost. In fact, this is often the case for an annealed specimen and, in consequence of this orientation, the cubes are withdrawn from planes parallel or at right angles to the metal surface. The random removal process leads to the production of a cavity having a cubic wall structure. When the selective attack occurs, tunnels branch outwards from the cavity surface. As a general 

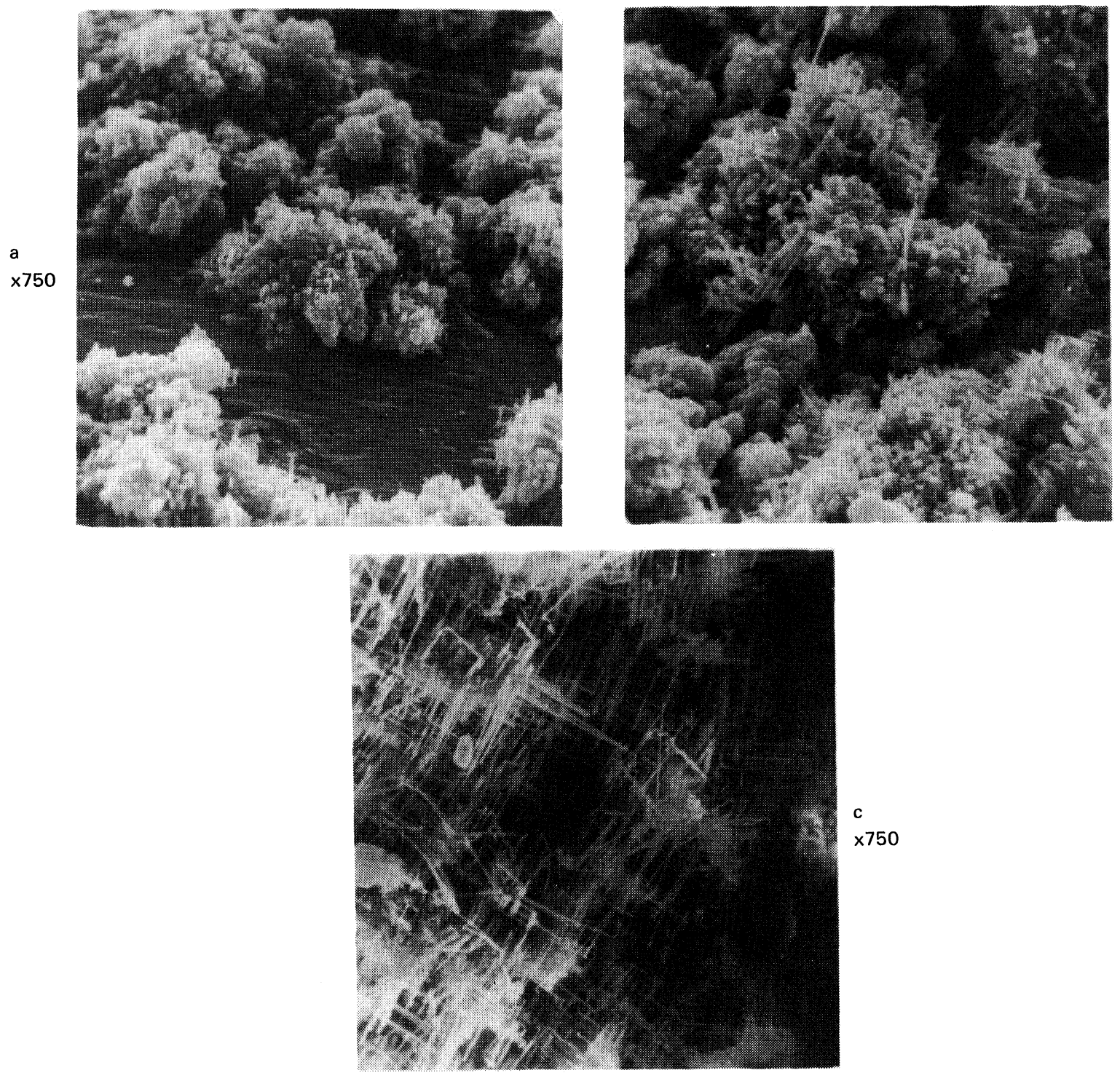

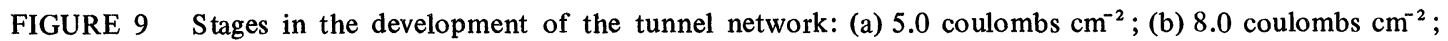
(c) 36 coulombs $\mathrm{cm}^{-2}$.

rule, both the random and the selective attack occur simultaneously.

In practice, it is desirable to minimise the random attack, since surface area increases associated with cavity formation are relatively small and excessively deep cavities may lead to a weakening of the foil. It is found that the dominance of one or the other of the two dissolution modes is almost entirely dependent upon the electrochemical parameters. The metallurgical characteristics of the foil appear to have very little influence. The way in which the electrochemical parameters affect tunnelling is summarised in
Table II. As the chloride concentration, the current density or the temperature increases, the tunnels become smaller in width and greater in numbers. These effects are illustrated in Figure 11, in which parametric values are high unless otherwise stated. The influence of increased current density is partly related to an increase in the number of active sites. It appears that the weak points in the surface oxide have a range of activities which are stimulated by raising the current density. Additives, both ionising and non-ionising, to the electrolyte can have important effects. A typical additive used commer- 


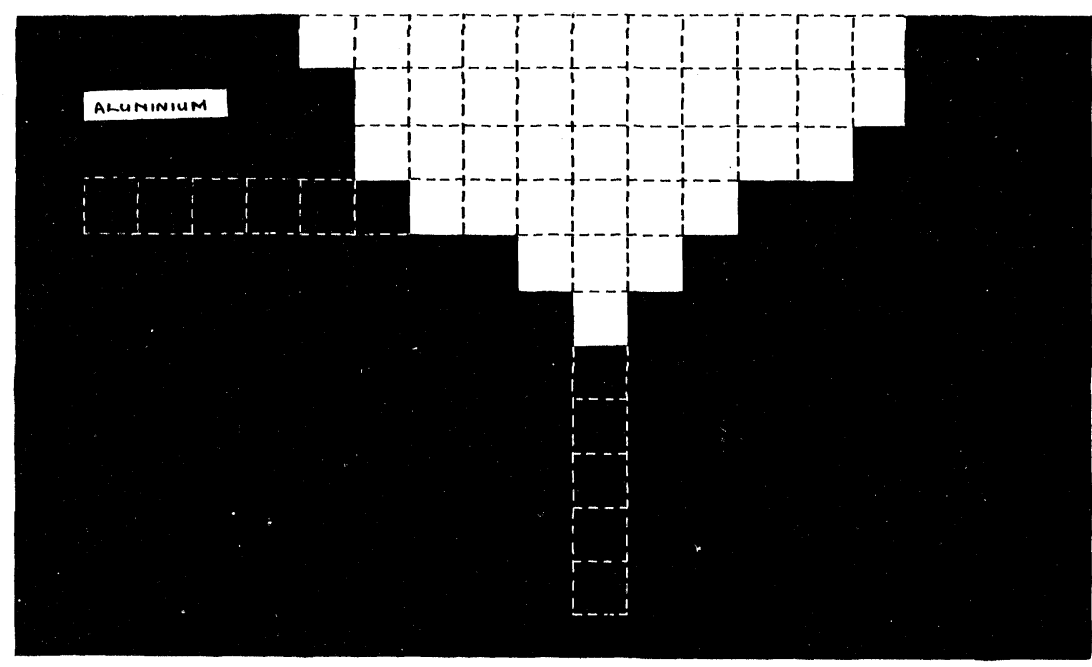

FIGURE 10 Schematic representation of the cube withdrawal model.

TABLE II

Influence of electrochemical parameters on degree of tunnelling

\begin{tabular}{ll}
\hline Parameter & Effect \\
\hline $\mathrm{Cl}^{-}$concentration & Increasing $\mathrm{Cl}^{-}$increases tunnelling \\
Current density & Increasing c.d. increases tunnelling \\
Temperature & Increasing $\mathrm{T}$ increases tunnelling \\
Additives & Not predictable, most have some effect \\
\hline
\end{tabular}

cially is sodium sulphate, which leads to a greater proliferation of narrower tunnels. Chromic oxide has a similar effect but additions of sodium nitrate lead to larger and less numerous tunnels. A common feature of all these effects is that a reduction in tunnel width is associated with a decrease in the size of the cubes and an increase in the number of tunnels.

Experiments indicate that similar effects are produced in bromide and iodide electrolytes. However, the degree of tunnelling and the surface gains achieved are lower than those found with chloride electrolytes. In consequence, no practical advantage is foreseen in using halides other than chloride or mixed halide electrolytes.

Of all the parameters controlling the etching process, temperature has the greatest effect. Regardless of all other factors, if the temperature is high some tunnelling is always observed. Temperature also appears to have a more direct influence on the cube size than any other parameter. Although there is a distribution in cube (or tunnel size) at each temperature, it is possible to estimate an average size ${ }^{6}$ and this is shown plotted against temperature in Figure 12. The logarithm of the cube size is found to diminish linearly with temperature and there is a fairly sharp differentiation between regions of tunnelling and those in which only cavity formation occurs. Thus below $60^{\circ} \mathrm{C}$ the cubes are relatively large and no tunnelling is observed. At $60^{\circ} \mathrm{C}$, incipient tunnelling is just apparent and at $80^{\circ} \mathrm{C}$ and $100^{\circ} \mathrm{C}$ tunnelling becomes widespread. This observation suggests the concept of a critical cube size at about $2 \mu \mathrm{m}$, above which no tunnelling can occur.

\section{DISCUSSION}

A linear relationship has been established between $E_{\mathrm{pit}}$ and the logarithm of the chloride ion concentration. A similar relationship was observed by Lepin and $\mathrm{Kadek}^{7}$ for the electrode potential of specimens of $99.5 \%$ aluminium immersed without polarisation in potassium chloride solution. They obtained $\epsilon=\epsilon_{0}+\mathrm{K} \log \mathrm{C}_{\mathrm{KC} 1}$ where $\epsilon_{0}$ was $-500 \mathrm{mV}$ at $20^{\circ} \mathrm{C}$ and $-520 \mathrm{mV}$ at $30^{\circ} \mathrm{C}$ and $\mathrm{K} \sim 60-70 \mathrm{mV}$. Other workers have determined individual values of $E_{\mathrm{pit}}$ for aluminium in sodium chloride solution using the potentiostatic method. In particular, Kaesche ${ }^{8}$ obtained $-0.48 \mathrm{~V}\left(1.0 \mathrm{M} \mathrm{NaCl}\right.$ at $\left.25^{\circ} \mathrm{C}\right)$ and Broli and $\mathrm{Holtan}^{2}-0.52 \mathrm{~V}\left(3 \% \mathrm{NaCl}\right.$ at $\left.30^{\circ} \mathrm{C}\right)$. Kaesche demonstrated that $E_{\text {pit }}$ was essentially independent of the $\mathrm{pH}$ of the sodium chloride solution in the range $\mathrm{pH} 2-11$. This result is important since it is evident that the $\mathrm{pH}$ within the surface pits decreases during etching in consequence of the partial hydrolysis of aluminium ions. As a result, a small amount of chemical dissolution occurs concomitantly with the electrochemical reaction and it is common 

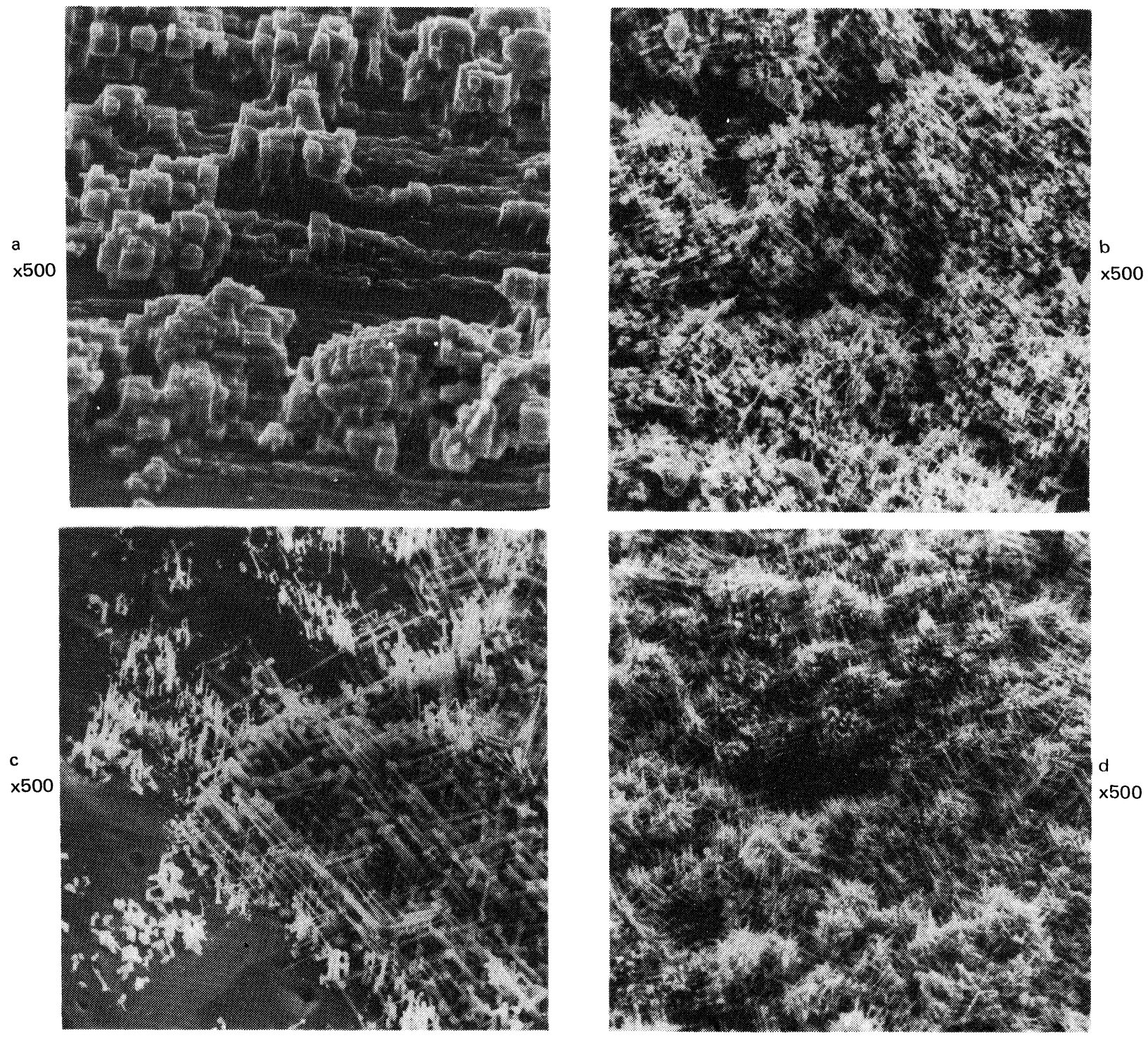

FIGURE 11 The influence of the electrochemical parameters on structure: (a) Low temperature; (b) Low concentration; (c) Low current density; (d) High temperature, concentration and current density.

experience that the weight loss of aluminium exceeds the value calculated from Faraday's Law.

No precise correlation has been achieved between the experimental $E_{0}$ and the calculated value based on the reaction given in Eq. (2). A similar disparity of about $0.3 \mathrm{~V}$ is observed for each of the three systems studied. An approximate correction introduced into the calculated value to allow for the possible amorphous structure of the aluminium halide resulted in only a small reduction in the observed inequality. However, possible alternative reactions leading to the formation of oxychlorides seem most unlikely in view of the free energies involved.

The directional nature of the dissolution appears to be related to the lower energy required to remove an atom from the $\{100\}$ plane. The phenomenon of crystallographic etching is not unusual and has been observed with other metals. ${ }^{9}$ Therefore, the random attack leading to the formation of surface cavities is readily explicable, although it is not clear why the dissolution should resemble a cube withdrawal mechanism. On the other hand, the selective attack 


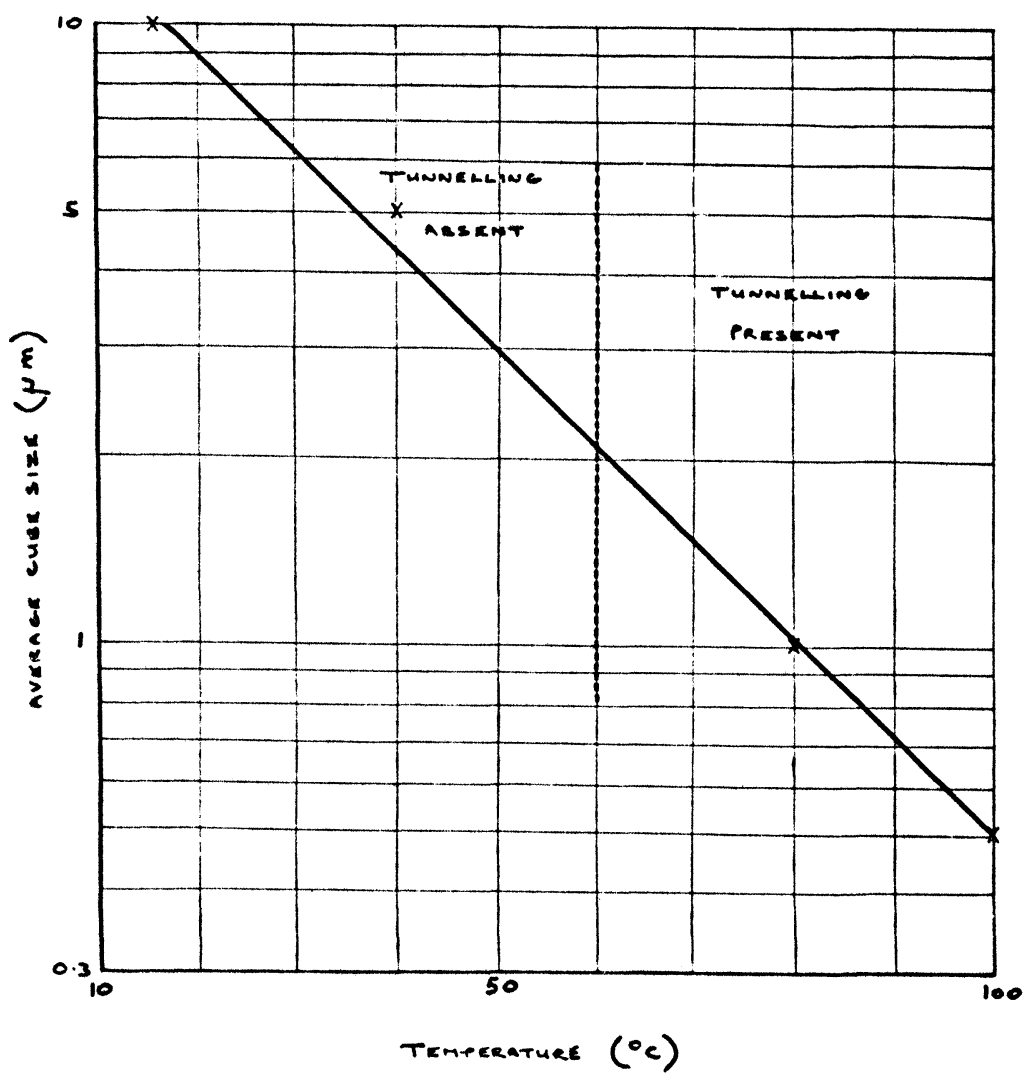

FIGURE 12 Variation of average cube size with temperature $\left(3.42 \mathrm{M}\right.$ sodium chloride, $0.6 \mathrm{~A} \mathrm{~cm}^{-2}, 24$ coulombs $\mathrm{cm}^{-2}$ ).

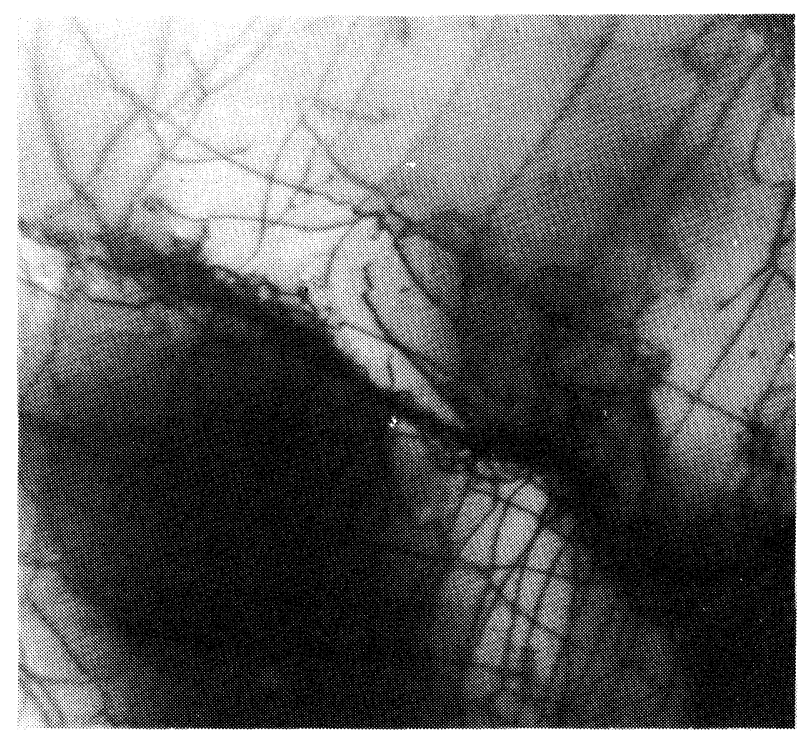

$\times 30000$

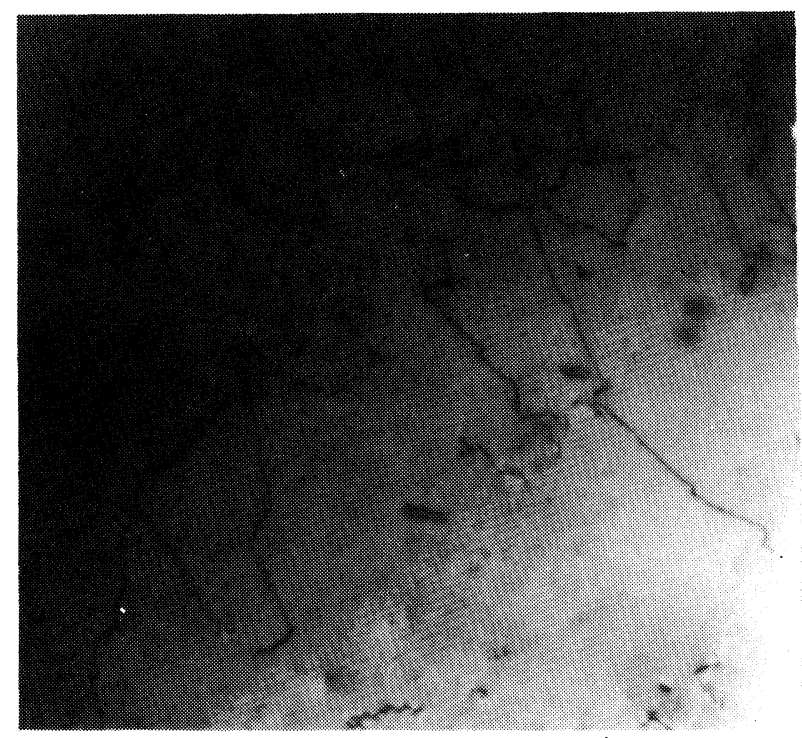

b

x30000

FIGURE 13 Dislocations in aluminium foil: (a) Surface; (b) Interior. 
leading to tunnelling is more difficult to understand. For example, studies do not indicate that there is a direct connection with dislocations, since the configurations of the latter are different in both scale and geometry (Figure 13). Equally, the presence of a basic dendritic structure within the metal resulting from constitutional supercooling in the original ingot seems remote, in view of the subsequent rolling and annealing necessary to prepare the foil. In the light of the strong dependence upon the electrochemical conditions, it seems that an explanation for tunnelling must be sought in electrochemical terms. Thus, it is considered that the underlying reason for this dependence is most likely to be connected with changes in electrolyte concentration, an effect that was thought to have caused the hysteresis in the potentiodynamic work. Furthermore, it is apparent that tunnels tend to narrow along their length (Figure 1(b)) an observation pointing to a progressive increase in chloride ion concentration. Therefore, taking into account the restricted diffusion path associated with tunnelling, it seems very possible that a high concentration of chloride ions could build up at the active face of the attack, i.e. at the end of the tunnel, and lower concentrations pertain down the major part of the tunnel. In this situation, the applied potential along the walls of the tunnel could fall below the pitting potential, resulting in the walls remaining passive while dissolution persists at the end face. Therefore, once a tunnel starts to form, the electrochemical conditions might be expected to favour a continuation of the selective attack over a more random one. Such a mechanism may also suggest a direct connection between cube size and tunnelling. For example, if the cube size in a surface cavity is relatively large, a concentration gradient sufficient to passivate the walls of the cube may be unable to form. Under these circumstances, the random attack may persist and the cavity continue to enlarge. Only when the cubes are of a certain size, i.e. the critical cube size, would tunnelling start.

It is evident that further work is necessary to establish the type of mechanism discussed above and, in particular, to assess the influence of other electrochemical parameters on the possible formation of concentration gradients within the tunnel network.

\section{CONCLUSIONS}

1) There is a linear relationship between $E_{\mathrm{pit}}$ and the logarithm of the chloride ion concentration.

2) The initial attack on the aluminium occurs at imperfections in the surface oxide film.

3) The subsequent dissolution of the metal can be likened to the removal of small cubes. The cubes may be taken from the metal either in a random way, producing surface cavities, or in a selective way, producing tunnels.

4) The size of the cubes and the predominance of either the random or the selective dissolution is primarily dependent upon the electrochemical parameters.

\section{ACKNOWLEDGEMENTS}

The author thanks Mr. N. S. Griffin for the scanning electron micrographs and Mr. R. Ogden for the transmission micrographs. This work is published by permission of The Plessey Company Limited

\section{REFERENCES}

1. H. Böhni and H. H. Uhlig, J. Electrochem. Soc. 116, 906 (1969).

2. A. Broli and H. Holtan, Corros. Sci. 13, 237 (1973).

3. N. F. Jackson, Component Technology 3, 11 (1968).

4. C. G. Dunn and R. B. Bolan, J. Electrochem. Soc. 116 , 1050 (1969).

5. C. G. Dunn, R. B. Bolan, A. S. Alwan and A.W. Stirling, J Electrochem. Soc. 118, 381 (1971).

6. A. R. Morley and D. S. Campbell, Radio \& Electron. Eng. 7, 421 (1973).

7. L. Lepin and V. Kadek, Corros. Sci. 6, 177 (1966).

8. H. Kaesche, Z. Phys. Chem., Frankfurt 34, 87 (1962).

9. M. J. Hill, J. Electrochem. Soc. 120, 142 (1973). 

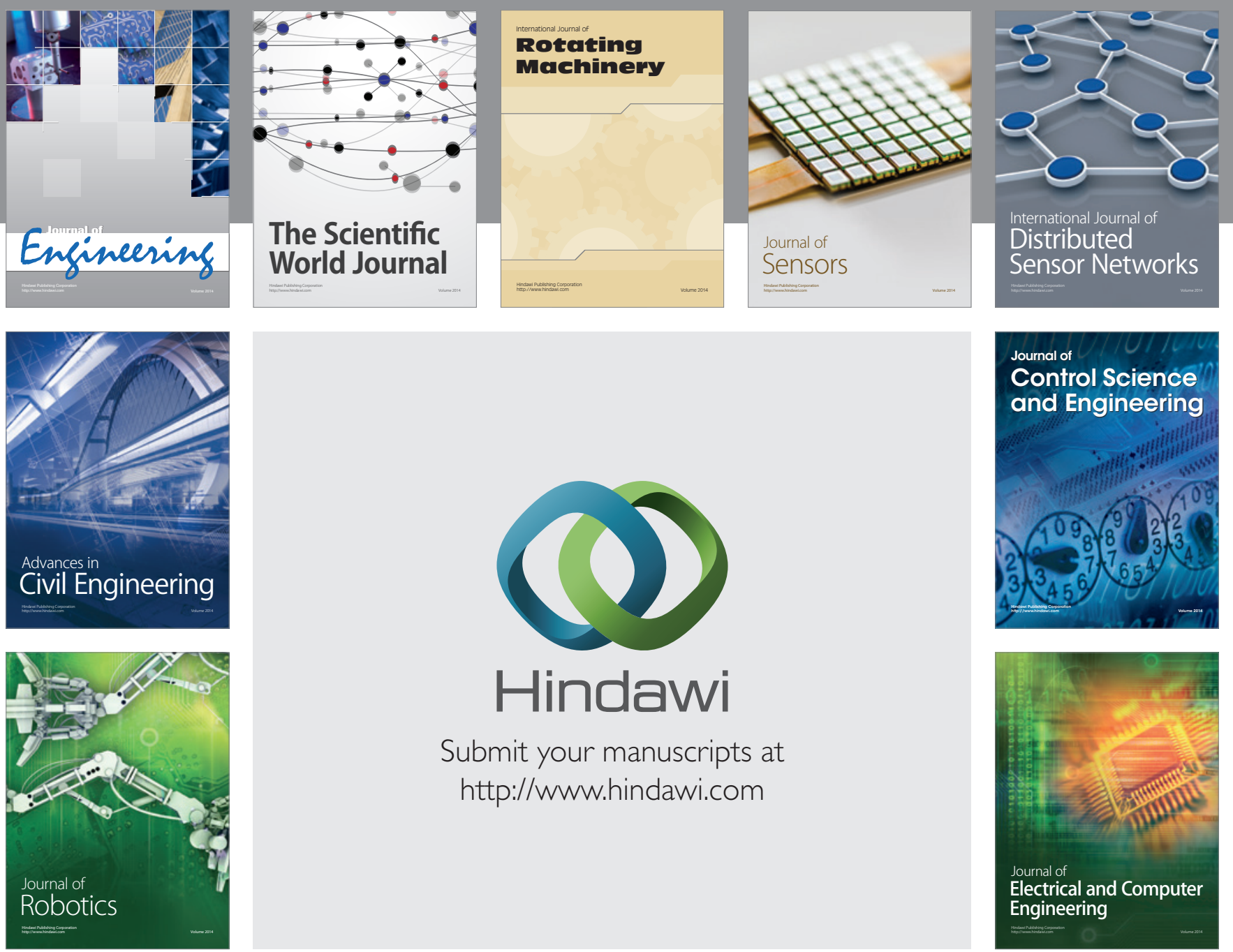

Submit your manuscripts at

http://www.hindawi.com
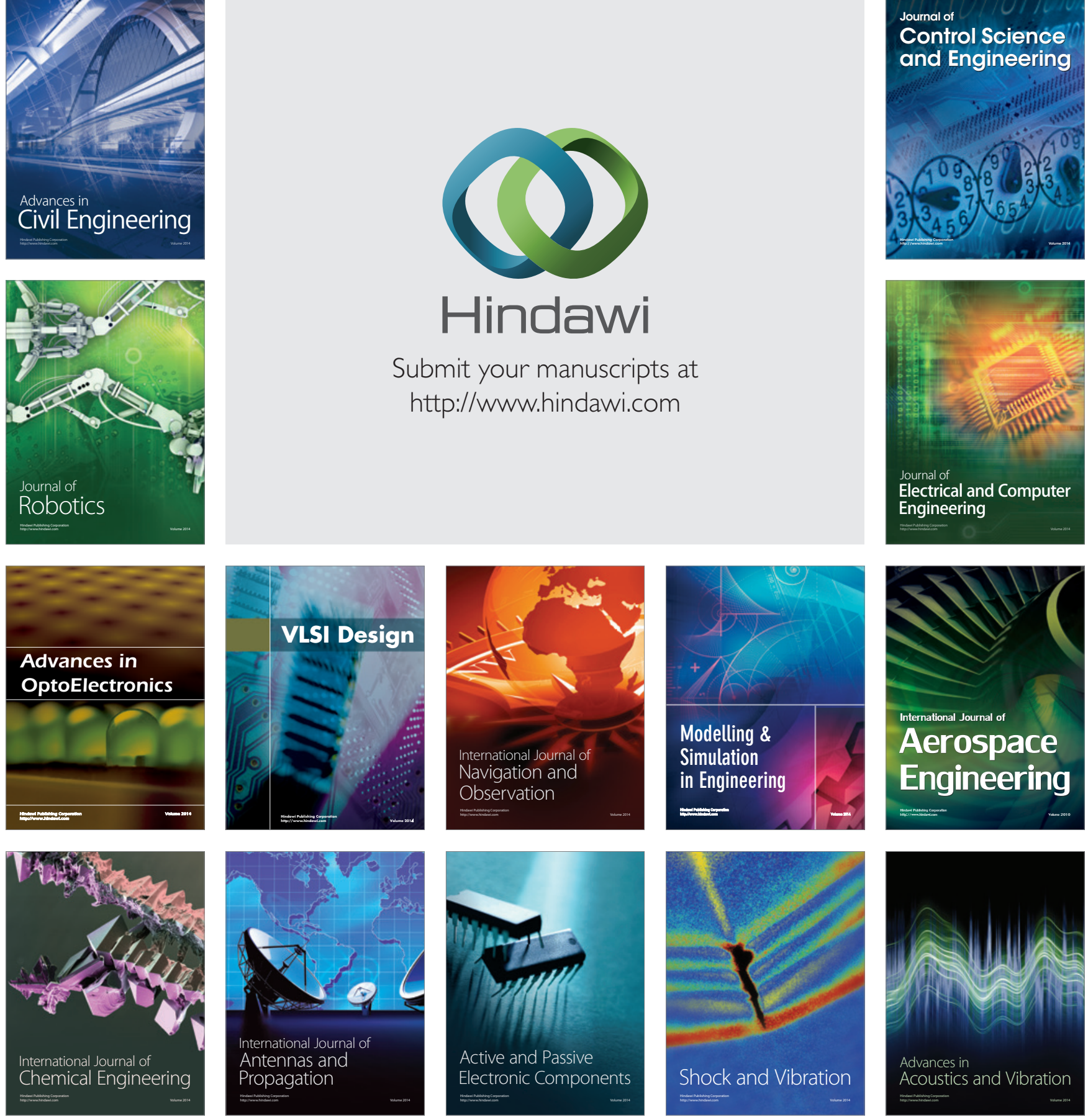